Check for updates

Cite this: J. Mater. Chem. A, 2020, 8 , 5750

Received 22nd January 2020

Accepted 2nd March 2020

DOI: $10.1039 / d 0 t a 00903 b$

rsc.li/materials-a

\section{Ultrahigh discharge efficiency and improved energy density in rationally designed bilayer polyetherimide- $\mathrm{BaTiO}_{3} / \mathrm{P}$ (VDF-HFP) composites $\uparrow$}

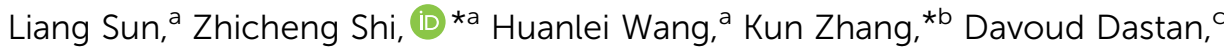 \\ Kai Sun ${ }^{d}$ and Runhua Fan ${ }^{d}$
}

Polymer dielectric composites are of great interest as film capacitors that are widely used in pulsed power systems. For a long time, huge efforts have been devoted to achieving energy densities as high as possible to satisfy the miniaturization and high integration of electronic devices. However, the discharge efficiency which is particularly crucial to practical applications has gained little attention. With the target of achieving concurrently improved energy density and efficiency, a class of rationally designed bilayer composites consisting of a pure polyetherimide layer and a $\mathrm{BaTiO}_{3} / \mathrm{P}(\mathrm{VDF}-\mathrm{HFP})$ composite layer were prepared. Interestingly, the bilayer composites exhibit ultrahigh discharge efficiencies $\eta$ (>95\%) under external electric fields up to $400 \mathrm{kV} \mathrm{mm}^{-1}$ which are much higher than most of the so far reported results $(\eta<80 \%$ ). Meanwhile, a low loss ( $\tan \delta<0.05$ a $10 \mathrm{kHz}$ ) comparable to that of the pure polyetherimide is obtained. In addition, the bilayer composites show impressive improvements in breakdown strengths $E_{\mathrm{b}}$, i.e., $285 \%, 363 \%, 366 \%$ and $567 \%$ for composites with 5 vol\%, 10 vol\%, 20 vol\% and 40 vol\% $\mathrm{BaTiO}_{3}$, compared to their single layer counterparts, resulting in obviously improved energy densities $U_{\mathrm{d}}$. In particular, the bilayer composite with 10 vol\% $\mathrm{BaTiO}_{3}$ displays the most prominent comprehensive energy storage performance, i.e., $\eta \sim 96.8 \%$ a $450 \mathrm{kV} \mathrm{mm} \mathrm{m}^{-1}, U_{\mathrm{d}} \sim 6 \mathrm{~J} \mathrm{~cm}^{-3}$ a $450 \mathrm{kV}$ $\mathrm{mm}^{-1}, \tan \delta \sim 0.025$ a $10 \mathrm{kHz}$, and $E_{\mathrm{b}} \sim 483.18 \mathrm{kV} \mathrm{mm}^{-1}$. The ultrahigh discharge efficiencies and high energy densities, along with low loss and breakdown strengths, make these bilayer composites ideal candidates for high-performance dielectric energy-storage capacitors.

\section{Introduction}

Polymer film capacitors (PFCs) have drawn considerable attention in recent years owing to their superior charge-discharge capabilities, outstanding cycling stabilities, excellent selfhealing capability and wide application in hybrid electric vehicles, medical defibrillators, electromagnetic launch systems, etc. ${ }^{1-4}$ However, the applications of PFCs are greatly restricted by their low energy densities. In principle, the energy density $\left(U_{\mathrm{d}}\right)$ of a dielectric material can be expressed by the equations $U_{\mathrm{d}}=$ $1 / 2 \varepsilon_{0} \varepsilon_{\mathrm{r}} E^{2}$ for a linear dielectric material and $U_{\mathrm{d}}=\int E \mathrm{~d} D$ for a nonlinear dielectric material, where $\varepsilon_{0}$ and $\varepsilon_{\mathrm{r}}$ represent the

${ }^{a}$ School of Materials Science and Engineering, Ocean University of China, Qingdao 266100, P. R. China. E-mail: zcshi@ouc.edu.cn

${ }^{b}$ Key Laboratory of Microgravity (National Microgravity Laboratory), Institute of Mechanics, Chinese Academy of Sciences, Beijing 100190, China. E-mail: zhangkun@imech.ac.cn

'School of Materials Science and Engineering, Georgia Institute of Technology, Atlanta, Georgia-30332, USA

${ }^{d}$ Institute of Marine Materials Science and Engineering, Shanghai Maritime University, Shanghai 201306, P. R. China

$\dagger$ Electronic supplementary information (ESI) available. See DOI: 10.1039/d0ta00903b dielectric permittivities of the vacuum and dielectrics, $E$ is the applied electric field which should be lower than the breakdown strength $\left(E_{\mathrm{b}}\right)$ of the materials, and $D=\varepsilon_{0} \varepsilon_{\mathrm{r}} E$ is the electric displacement. Accordingly, high $\varepsilon_{\mathrm{r}}$ and high $E_{\mathrm{b}}$ are desired for high $U_{\mathrm{d}}$. To achieve this, various strategies have been proposed, among which constructing polymer based composites filled with high $\varepsilon_{\mathrm{r}}$ or high $E_{\mathrm{b}}$ fillers has been demonstrated to be effective. ${ }^{5-7}$ To obtain improved $\varepsilon_{\mathrm{r}}$, ferroelectric ceramic fillers (e.g., $\mathrm{BaTiO}_{3}{ }^{\mathbf{8}, \mathbf{9}} \mathrm{SrTiO}_{3},{ }^{10} \mathrm{NaNbO}_{3},{ }^{11}$ etc.) and conductors (e.g., metals, ${ }^{12-14}$ carbon nanotubes,${ }^{15}$ graphene, ${ }^{16,17}$ conductive polymers, ${ }^{18}$ etc.) are usually employed. However, improved $\varepsilon_{\mathrm{r}}$ is always accompanied by suppressed $E_{\mathrm{b}}$, deteriorated $\eta$ and elevated loss. ${ }^{19}$ To achieve improved $E_{\mathrm{b}}$, fillers with high $E_{\mathrm{b}}(e . g$., boron nitride, alumina, silica, etc.) are usually employed., Unfortunately, the fillers with high $E_{\mathrm{b}}$ often exhibit low $\varepsilon_{\mathrm{r}}$, leading to suppressed $\varepsilon_{\mathrm{r}}{ }^{20}$ As a result, a reasonable balance between $\varepsilon_{\mathrm{r}}$ and $E_{\mathrm{b}}$ has been a problem demanding a prompt solution until now. Although various innovative strategies, such as designing core-shell structured fillers ${ }^{21,22}$ and surface modification of fillers, ${ }^{23-25}$ have been proposed to address this dilemma, the effect is still not satisfactory.

Recently, researchers found that multilayer structured composites may offer a feasible paradigm to realize the 
concurrent improvement of $\varepsilon_{\mathrm{r}}$ and $E_{\mathrm{b}}{ }^{26-29}$ Compared with singlelayer composites, multilayer composites possess another type of mesoscopic interface between adjacent layers, in addition to the microscopic interfaces between the filler and matrix, which may induce strong interfacial polarization and extra charge storage. ${ }^{30-32}$ Wang and co-workers reported the design of trilayered films consisting of stacked barium titanate/poly(vinylidene fluoride-co-hexafluoropropylene $) \quad\left(\mathrm{BaTiO}_{3} / \mathrm{P}(\mathrm{VDF}-\mathrm{HFP})\right)$ nanocomposite layers, where the middle layer with a high $\mathrm{BaTiO}_{3}$ content offered high $\varepsilon_{\mathrm{r}}$ while the two outer layers with a low $\mathrm{BaTiO}_{3}$ content provided high $E_{\mathrm{b}}$. A significantly improved $E_{\mathrm{b}}$ of $\sim 526 \mathrm{kV} \mathrm{mm}^{-1}$ and a moderately enhanced $\varepsilon_{\mathrm{r}}$ of $\sim 12$ @ $1 \mathrm{kHz}$, in comparison with those of pure $\mathrm{P}(\mathrm{VDF}-\mathrm{HFP})\left(E_{\mathrm{b}}=410 \mathrm{kV} \mathrm{mm}{ }^{-1}\right.$ and $\varepsilon_{\mathrm{r}}=10$ @ $1 \mathrm{kHz}$ ), are realized via controlling the $\mathrm{BaTiO}_{3}$ content. Consequently, an ultrahigh discharge energy density of $26.4 \mathrm{~J} \mathrm{~cm}^{-3}$ and a superior discharge efficiency of $72 \%$ are obtained. ${ }^{33}$ In addition to the typical trilayered composites, much attention has also been paid to multilayer composites with more than three layers. Jiang and co-workers ${ }^{27}$ designed a series of multilayer $\mathrm{BaTiO}_{3} / \mathrm{P}(\mathrm{VDF}-\mathrm{HFP})$ composites with different numbers of layers. It was shown that a composite with 16 layers exhibited a substantially improved $E_{\mathrm{b}}\left(\sim 862.5 \mathrm{kV} \mathrm{mm}^{-1}\right)$ and an obviously enhanced $\varepsilon_{\mathrm{r}}(\sim 16$ @ $1 \mathrm{kHz})$ compared with pure $\mathrm{P}(\mathrm{VDF}-$ HFP) $\left(E_{\mathrm{b}}=600 \mathrm{kV} \mathrm{mm}{ }^{-1}, \varepsilon_{\mathrm{r}}=10\right.$ @ $\left.1 \mathrm{kHz}\right)$. Therefore, an ultrahigh energy density of $\sim 30.15 \mathrm{~J} \mathrm{~cm}^{-3}$ and a high discharge efficiency of $\sim 78 \%$ were achieved simultaneously.

Up to now, numerous multilayer composites with rationally designed hierarchical structures have been developed. ${ }^{33-37}$ However, most of the studies aimed at achieving energy densities as high as possible and thus the nonlinear ferroelectric polymer poly(vinylidene fluoride) and its copolymers were chosen as the matrix. Although greatly improved energy densities have been obtained, it inevitably results in a suppressed discharge efficiency $(\eta<80 \%)$ and elevated loss ( $\tan \delta>0.1$ @ 1
$\mathrm{kHz}$ ), which cannot meet the requirements of practical applications. In other words, more attention should be paid to the concurrent improvement of $U_{\mathrm{d}}$ and $\eta$ rather than solely $U_{\mathrm{d}}$. As we know, linear dielectric polymers (e.g., polyetherimide, polypropylene, polyimide, and polymethyl methacrylate) have a very high $\eta(>95 \%)$ and low loss $(\tan \delta<0.05$ @ $1 \mathrm{kHz}) .{ }^{2,19}$ With the aim of achieving balanced energy density and discharge efficiency, we herein report a novel design of bilayer dielectric composites consisting of a pure polyetherimide (PEI) layer and a $\mathrm{BaTiO}_{3} / \mathrm{P}(\mathrm{VDF}-\mathrm{HFP})$ composite layer. In comparison with a trilayer composite which has two layer/layer interfaces, a bilayer composite possesses only one layer/layer interface. Therefore, the loss induced by the interfacial polarization at the interfaces between adjacent layers could be suppressed in the bilayer composite, which is favorable for the achievement of high efficiency and low loss. As shown in Fig. 1, the linear dielectric layer PEI offers high efficiency $\eta$, low loss, and high breakdown strength $E_{\mathrm{b}}$, while the nonlinear dielectric $\mathrm{BaTiO}_{3} /$ $\mathrm{P}\left(\right.$ VDF-HFP) composite layer provides high energy density $U_{\mathrm{d}}$. Consequently, the bilayer composites may combine the superiorities of the two layers, yielding simultaneously high $\eta$, high $U_{\mathrm{d}}$, high $E_{\mathrm{b}}$, and low loss. Interestingly, our results show that all of the bilayer composites exhibit ultrahigh $\eta$ values over 95\% which are much higher than those of most of the so far reported polymer dielectric composites $(\eta<80 \%)$ under external electric fields up to $400 \mathrm{kV} \mathrm{mm}^{-1}$. Moreover, a low loss ( $\tan \delta<0.05$ @ $10 \mathrm{kHz}$ ) comparable to that of the pure PEI is also achieved. Furthermore, the bilayer composites show impressive improvements in $E_{\mathrm{b}}$, i.e., $285 \%, 363 \%, 366 \%$ and $567 \%$ for composites with 5 vol\%, $10 \mathrm{vol} \%, 20 \mathrm{vol} \%$ and $40 \mathrm{vol} \% \mathrm{BaTiO}_{3}$, compared to their single-layer counterparts, thus resulting in obviously improved $U_{\mathrm{d}}$. The outstanding dielectric characteristics make these bilayer composites promising candidates for high-performance energy-storage capacitors.

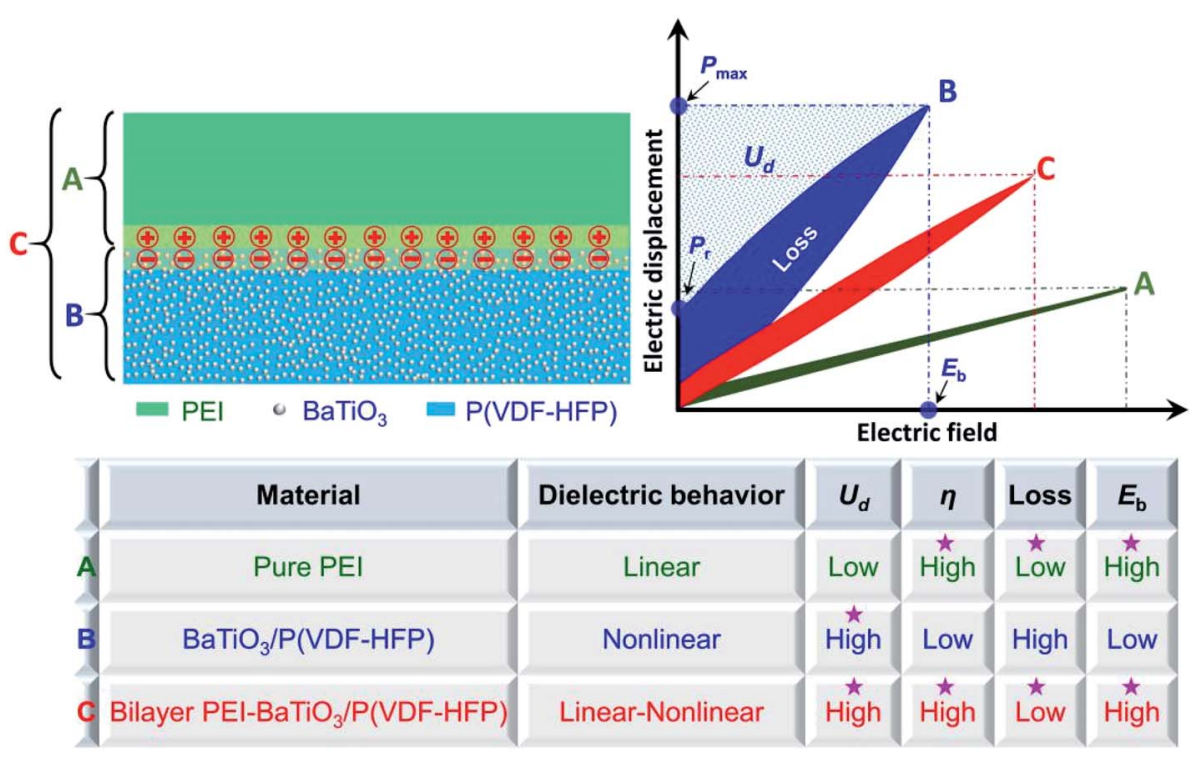

Fig. 1 Schematic illustration of the dielectric energy-storage characteristics of linear dielectric, nonlinear dielectric and bilayer linear/nonlinear dielectric composites. The pentagrams indicate the desired properties. 


\section{Experimental}

\subsection{Materials}

Barium titanate $\left(\mathrm{BaTiO}_{3},<3 \mu \mathrm{m},>99.5 \%\right.$, Aladdin Industrial Corporation), poly(vinylidene fluoride-hexafluoropropylene) (P(VDF-HFP), 15\% HFP, PolyK Technologies, USA), polyetherimide (PEI, PolyK Technologies, USA), 1-methyl-2pyrrolidone $\left(\mathrm{C}_{5} \mathrm{H}_{9} \mathrm{NO}, \geq 99.0 \%\right.$ Sinopharm Chemical Reagent Co., Ltd.), sodium dodecylbenzenesulfonate $\left(\mathrm{C}_{18} \mathrm{H}_{29} \mathrm{NaO}_{3}\right.$, $\geq 88.0 \%$, Sinopharm Chemical Reagent Co., Ltd.) and ethanol ( $\geq 99.7 \%$, Sinopharm Chemical Reagent Co., Ltd.).

\subsection{Preparation of bilayer composites}

Firstly, a $\mathrm{BaTiO}_{3} / \mathrm{P}(\mathrm{VDF}-\mathrm{HFP})$ suspension and PEI solution were prepared. For the preparation of the $\mathrm{BaTiO}_{3} / \mathrm{P}(\mathrm{VDF}-\mathrm{HFP})$ suspension, $\mathrm{BaTiO}_{3}$ particles and sodium dodecylbenzenesulfonate (SDBS) were ultrasonically dispersed into $10 \mathrm{~mL} \mathrm{1-}$ methyl-2-pyrrolidone (NMP) for $1 \mathrm{~h}$ at room temperature to obtain a stable suspension, followed by adding P(VDF-HFP) pellets into the suspension with magnetic stirring at $75{ }^{\circ} \mathrm{C}$ until the $\mathrm{P}(\mathrm{VDF}-\mathrm{HFP})$ pellets were dissolved completely. For the preparation of the PEI solution, PEI particles were directly dissolved in 1-methyl-2-pyrrolidone (NMP) with rapid magnetic stirring at $75{ }^{\circ} \mathrm{C}$ for $5 \mathrm{~h}$ and mild stirring at room temperature overnight. Then the $\mathrm{BaTiO}_{3} / \mathrm{P}(\mathrm{VDF}-\mathrm{HFP})$ suspension and PEI solution were cast on glass plates separately. The thicknesses of the single-layer composites were controlled in the range of 15$20 \mu \mathrm{m}$ by adjusting the height of blade. Then the films along with the glass plates were transferred to an oven and dried at $100{ }^{\circ} \mathrm{C}$ for $4 \mathrm{~h}$ and $200^{\circ} \mathrm{C}$ for $5 \mathrm{~min}$. Subsequently, the films were peeled off via quenching in ice water and dried in an oven at $70{ }^{\circ} \mathrm{C}$ for $6 \mathrm{~h}$, yielding the single layer $\mathrm{BaTiO}_{3} / \mathrm{P}(\mathrm{VDF}-\mathrm{HFP})$ composite films and PEI films. Finally, bilayer films with different $\mathrm{BaTiO}_{3}$ contents were obtained via stacking and hotpressing a $\mathrm{BaTiO}_{3} / \mathrm{P}(\mathrm{VDF}-\mathrm{HFP})$ composite film and a PEI film at $150{ }^{\circ} \mathrm{C}$ under a pressure of $6 \mathrm{MPa}$ for $5 \mathrm{~min}$. In this paper, the single layer $\mathrm{BaTiO}_{3} / \mathrm{P}(\mathrm{VDF}-\mathrm{HFP})$ composites and bilayer composites were denoted as $\alpha$ vol\% BT/PVDF and PEI- $\alpha$ vol\% $\mathrm{BT} / \mathrm{PVDF}$, respectively, where $\alpha$ represents the volume fraction of $\mathrm{BaTiO}_{3}$ in the $\mathrm{BaTiO}_{3} / \mathrm{P}(\mathrm{VDF}-\mathrm{HFP})$ composites.

\subsection{Characterization and measurements}

The morphologies of the bilayer films and elemental distributions of barium and carbon were observed by scanning electron microscopy (SEM, S-4800, Hitachi, Ltd.) combined with EDX. Circular gold electrodes with a diameter of $2.98 \mathrm{~mm}$ were sputtered on the two sides of the samples before dielectric measurements. The dielectric properties were analyzed with an Agilent E4980A Precision LCR analyzer in the frequency range from $100 \mathrm{~Hz}$ to $1 \mathrm{MHz}$. Open and short compensations were performed before testing. The permittivity was calculated using $\varepsilon_{\mathrm{r}}=t C_{\mathrm{p}} / A \varepsilon_{0}$, where $t$ is the thickness of the sample, $A$ is the area of the electrode, $C_{\mathrm{p}}$ is the parallel capacitance, $f$ is the electric field frequency, and $\varepsilon_{0}$ is the absolute permittivity of free space $\left(8.85 \times 10^{-12} \mathrm{~F} \mathrm{~m}^{-1}\right)$. The breakdown strengths were obtained using a setup equipped with a Trek 609A amplifier with a voltage ramping rate of $500 \mathrm{~V} \mathrm{~s}^{-1}$ at room temperature (PolyK Technologies, USA). The energy storage performances, including discharge energy densities and charge-discharge efficiencies, were obtained using $P-E$ hysteresis loops which were collected at $1 \mathrm{kHz}$ using a ferroelectric test system based on a modified Sawyer-Tower circuit (PolyK Technologies, USA).

\section{Results and discussion}

The optical photograph and cross-sectional SEM morphologies of the PEI-20 vol\% BT/PVDF bilayer composite are presented in Fig. 2a-d. As can be seen, the bilayer film is flexible and has smooth surfaces. The SEM image confirms the distinct bilayer configuration, in which the two layers are tightly welded together without the appearance of noticeable defects (e.g., pores, cracks, voids, etc.). Moreover, the high-magnification cross-sectional SEM images shown in Fig. S1† demonstrate that the $\mathrm{BaTiO}_{3}$ particles are homogeneously dispersed in the $\mathrm{P}(\mathrm{VDF}-\mathrm{HFP})$

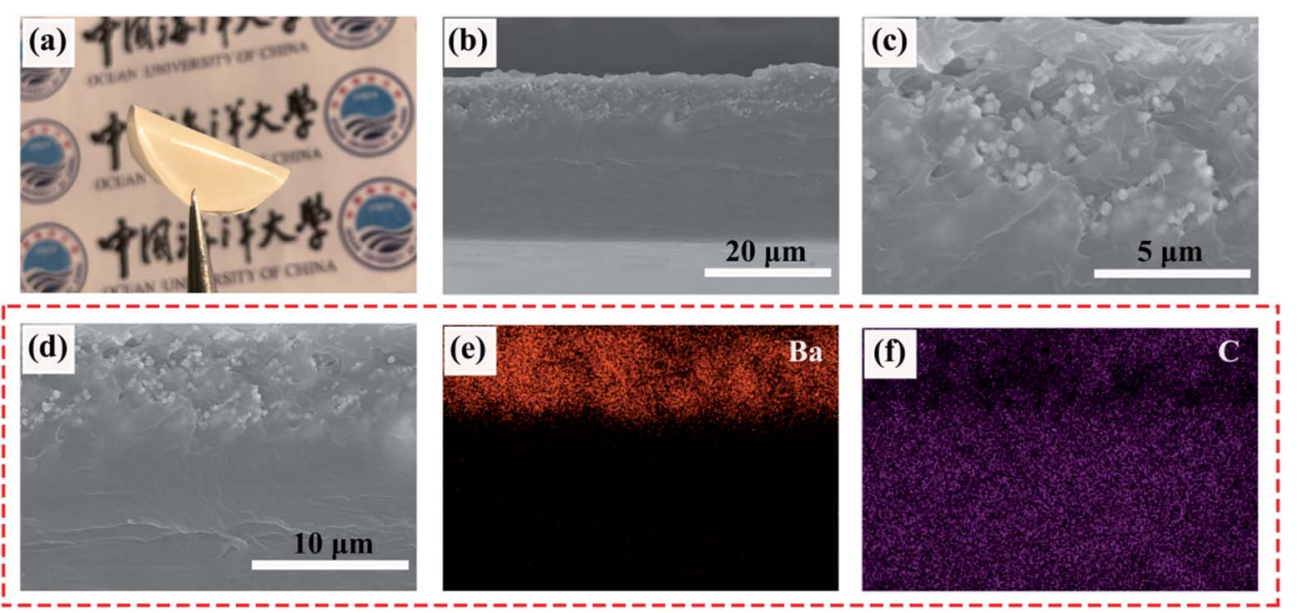

Fig. 2 (a) Photograph, (b-d) cross-sectional SEM morphologies and (e and f) corresponding EDX mapping images of the PEI-20 vol\% BT/PVDF bilayer composite. 
matrix. The EDX mapping results further demonstrate that the $\mathrm{BaTiO}_{3}$ particles are well restricted in the $\mathrm{P}(\mathrm{VDF}-\mathrm{HFP})$ matrix without apparent diffusion into the PEI layer. Furthermore, the total thickness of the film is $\sim 25 \mu \mathrm{m}$, where the BT/PVDF layer is $\sim 6.6 \mu \mathrm{m}$ while the PEI layer is $\sim 18.4 \mu \mathrm{m}$. As we know, in a singlelayer composite consisting of ferroelectric fillers (e.g., $\mathrm{BaTiO}_{3}$ ) dispersed in a linear dielectric matrix (e.g., PEI, PMMA, and PI), the ferroelectric fillers offer high energy-storage density while the matrix maintains the high energy-storage efficiency. As the loading fraction of ferroelectric fillers increases, the energy density increases and the efficiency decreases. ${ }^{38,39}$ Similarly, in the bilayer-structured composites, the linear dielectric PEI layer is expected to contribute to the improved energy-storage efficiency, while the ferroelectric $\mathrm{BaTiO}_{3} / \mathrm{P}(\mathrm{VDF}-\mathrm{HFP})$ layer could suppress the efficiency because of its strong polarization-induced energy loss. Therefore, to achieve high efficiency, the volume fractions of ferroelectric $\mathrm{BaTiO}_{3}$ and $\mathrm{P}(\mathrm{VDF}-\mathrm{HFP})$ in the bilayer composites should be lowered. In this regard, the PEI layer is designed to be much thicker than the $\mathrm{BaTiO}_{3} / \mathrm{P}(\mathrm{VDF}-\mathrm{HFP})$ layer. However, it should be noted that the increasing thickness ratio of the PEI layer to the $\mathrm{BaTiO}_{3} / \mathrm{P}(\mathrm{VDF}-\mathrm{HFP})$ layer would also result in suppressed energy-storage density. As shown in Fig. S1, $\uparrow$ the thickness ratios of the BT/PVDF layer to the PEI layer for all the bilayer composites are almost the same, i.e., $\sim 0.36$. Accordingly, the volume ratio of the BT/PVDF layer to the PEI layer is about 0.36 , and the actual volume fractions of $\mathrm{BaTiO}_{3}$ in the PEI-5 vol\% BT/PVDF, PEI-10 vol\% BT/PVDF, PEI-20 vol\% BT/PVDF and PEI$40 \mathrm{vol} \% \mathrm{BT} / \mathrm{PVDF}$ composites are $1.8 \%, 3.6 \%, 7.2 \%$ and $14.4 \%$, respectively.

The frequency dependences of dielectric permittivity and loss tangent for single layer and bilayer composites is displayed in Fig. 3. As shown in Fig. 3a, the pure PEI film shows the lowest permittivity and is almost independent of frequency in the tested frequency range. The pure $\mathrm{P}(\mathrm{VDF}-\mathrm{HFP})$ has a much higher permittivity than pure PEI and exhibits apparent frequency dispersion behavior. The permittivities of the $\mathrm{BaTiO}_{3} /$ $\mathrm{P}(\mathrm{VDF}-\mathrm{HFP})$ composites increase with higher $\mathrm{BaTiO}_{3}$ loadings, which could be attributed to the strong inherent dipolar polarization inside the ferroelectric $\mathrm{BaTiO}_{3}$ particles. In addition, the interfacial polarization of the accumulated charges at the interfaces between $\mathrm{BaTiO}_{3}$ particles and the P(VDF-HFP) matrix may also contribute to the enhanced dielectric permittivity. Correspondingly, the pure PEI film possesses the lowest loss, while the pure PVDF and $\mathrm{BaTiO}_{3} / \mathrm{P}(\mathrm{VDF}-\mathrm{HFP})$ composites exhibit much higher loss than PEI (see Fig. 3b). For instance, the loss tangents of the composites with $10 \mathrm{vol} \%$ and $20 \mathrm{vol} \%$ $\mathrm{BaTiO}_{3}$ come up to above 0.1 @ $10 \mathrm{kHz}$, which may result in a huge amount of undesired heat production, thus deteriorating the performance stability and shortening the service life of the electronic devices. As shown in Fig. 3c and d, the bilayer composites display moderate dielectric permittivities that are between those of the corresponding single layer $\mathrm{BaTiO}_{3} / \mathrm{P}(\mathrm{VDF}-$ HFP) composites and the pure PEI layer. Fortunately, low dielectric loss ( $\tan \delta<0.05$ @ $10 \mathrm{kHz}$ ) is achieved in the bilayer composites which will greatly facilitate their practical application.

It should be noted that the magnitude of dielectric permittivity can only determine the energy density of a dielectric material under a certain electric field. In addition to permittivity, the maximum electric field that a dielectric material can withstand before failure (i.e., breakdown strength $E_{\mathrm{b}}$ ) is another crucial parameter for dielectric energy-storage materials, (a)

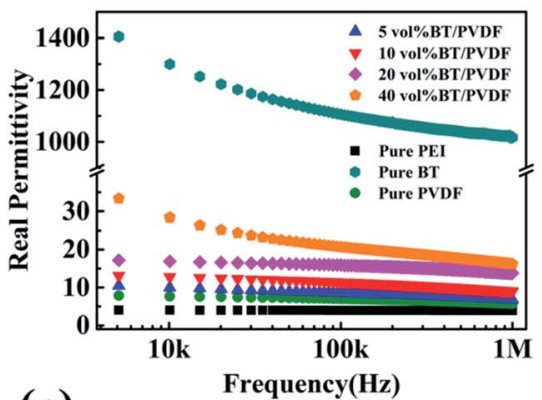

(c)

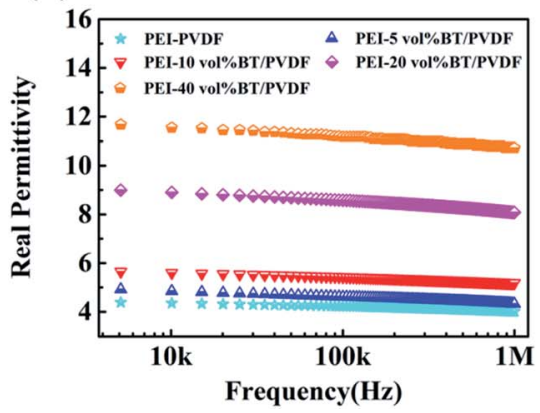

(b)

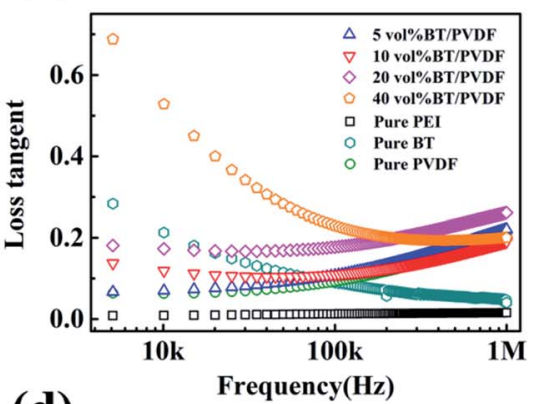

(d)

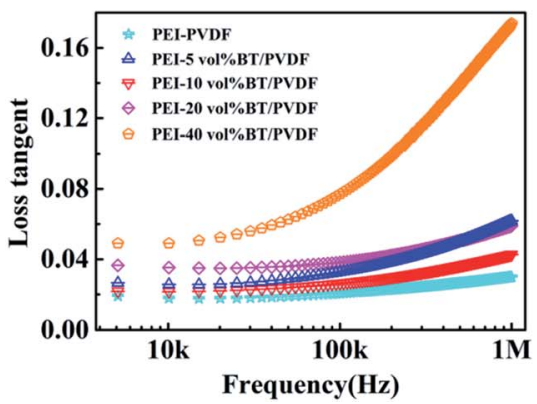

Fig. 3 Frequency dependences of dielectric permittivity and loss tangent for ( $a$ and b) single layer and (c and d) bilayer composites. 
especially for their applications under high electric fields. Therefore, the breakdown strengths of the composites are further evaluated using the two-parameter Weibull distribution, which is described by eqn (1):

$$
P(E)=1-\exp \left[-\left(\frac{E}{\alpha}\right)^{\beta}\right]
$$

where $P(E)$ is the cumulative failure probability, $E$ is the experimentally tested breakdown electric field, $\alpha$ is the electric field for which there is a $63.2 \%$ probability of sample breakdown (Weibull breakdown strength, $E_{\mathrm{b}}$ ), and $\beta$ is a shape parameter or the slope of the derived logarithm function reflecting the scatter of the tested $E_{\mathrm{b}}$. When the $\beta$ value is 3, the tested data follow Gaussian distribution, and a higher value of $\beta$ implies a higher level of reliability. Fig. 4 presents the Weibull $E_{\mathrm{b}}$ of the single layer and bilayer composites. We can see that all of the plots show high $\beta$ values, indicating high reliability of the measured data. The pure PEI has the highest $E_{\mathrm{b}}$ of $\sim 607.35 \mathrm{kV} \mathrm{mm}^{-1}$ and pure P(VDFHFP) also exhibits a high $E_{\mathrm{b}}$ of $\sim 463.90 \mathrm{kV} \mathrm{mm}^{-1}$. However, substantially deteriorated $E_{\mathrm{b}}$ values, which are only $\sim 35 \%$, $\sim 29 \%, 22 \%$ and $\sim 13 \%$ that of the pure P(VDF-HFP) matrix are observed in the single-layer $\mathrm{BT} / \mathrm{P}(\mathrm{VDF}-\mathrm{HFP})$ composites with $5 \mathrm{vol} \%, 10 \mathrm{vol} \%, 20 \mathrm{vol} \%$ and $40 \mathrm{vol} \% \mathrm{BaTiO}_{3}$, respectively. This is a widely reported phenomenon in ferroelectric ceramic/ polymer composites because of the severe electric field distortion in the region near the ceramic particles which facilitates the development of electric trees. ${ }^{27,30}$ It is worth noting that the bilayer composites show remarkably improved $E_{\mathrm{b}}$ compared with their single-layer counterparts. Specifically, the $E_{\mathrm{b}}$ values of the bilayer composites with $5 \mathrm{vol} \%, 10 \mathrm{vol} \%, 20 \mathrm{vol} \%$ and $40 \mathrm{vol} \%$ $\mathrm{BaTiO}_{3}$ are $\sim 285 \%, \sim 363 \%, 366 \%$ and $\sim 567 \%$ that of their single layer counterparts. In particular, the bilayer composite with 10 vol\% $\mathrm{BaTiO}_{3}$ exhibits an outstanding $E_{\mathrm{b}}$ of $483.18 \mathrm{kV} \mathrm{mm}^{-1}$, which is even higher than that of the pure $\mathrm{P}(\mathrm{VDF}-\mathrm{HFP})$. In the bilayer composites, although the $\mathrm{BT} / \mathrm{P}(\mathrm{VDF}-\mathrm{HFP})$ layer is easy to break down, the majority of the applied electric voltage will concentrate on the PEI layer which possesses high $E_{\mathrm{b}}$, thus relieving the electric field in the BT/P(VDF-HFP) layer. ${ }^{33-35}$ Moreover, the development of electric trees could be effectively blocked by the huge electric field gap between the two layers, thus preventing the electric trees from penetrating the entire bilayer composites. In addition, the electric trees may develop along the interfaces between the adjacent layers, yielding an increased length of electric tree development paths which is beneficial for the improvement of $E_{\mathrm{b}}$.

The unipolar electric displacement-electric field $(P-E)$ loops for the single layer and bilayer composites are illustrated in Fig. 5, S3 and S4. $†$ Slim (or narrow) loops imply a weak deviation from the linear dielectric behavior and correspond to a low energy loss. Clearly, the pure P(VDF-HFP) shows much wider loops than the pure PEI, and the introduction of $\mathrm{BaTiO}_{3}$ particles further widens the loops, indicative of enhanced energy loss and suppressed efficiency (see Fig. 5a). The bilayer composites possess slim loops located between those of the pure PEI and pure P(VDF-HFP), and no serious broadening phenomenon is observed in the loops under elevated electric fields (see Fig. $5 \mathrm{~b}$ and $\mathrm{S} 4 \dagger$ ). Fig. $5 \mathrm{c}$ depicts the displacements of the pure PEI and bilayer composites with varied $\mathrm{BaTiO}_{3}$ loading fractions at $350 \mathrm{kV} \mathrm{mm}^{-1}$. As can be see, the bilayer composites exhibit substantially improved $P_{\max }\left(>2 \mu \mathrm{C} \mathrm{cm}{ }^{-2}\right)$ compared with the pure PEI $\left(\sim 1.25 \mu \mathrm{C} \mathrm{cm}^{-2}\right)$ which is induced by the introduction of the ferroelectric $\mathrm{BT} / \mathrm{P}(\mathrm{VDF}-\mathrm{HFP})$ layer with strong dipolar and interfacial polarizations. In contrast, only slight increments of $P_{\mathrm{r}}\left(<0.25 \mu \mathrm{C} \mathrm{cm}{ }^{-2}\right)$ are observed, yielding an increased value of $\left(P_{\max }-P_{\mathrm{r}}\right)$ which is beneficial for the concurrent improvement of energy density and efficiency. Moreover, the $P_{\max }$ values of the composites become higher with increasing loading fractions of $\mathrm{BaTiO}_{3}$ which should be ascribed to the high $P_{\max }\left(\sim 0.085 \mathrm{C} \mathrm{m}^{-2}\right.$ at $\left.3 \mathrm{kV} \mathrm{mm}^{-1}\right)$ of pure $\mathrm{BaTiO}_{3}$ (see Fig. S3b $\dagger$ ). However, a higher $\mathrm{BaTiO}_{3}$ content also brings about enhanced deviations of $P-E$ loops from linear behavior, leading to suppressed energy-storage performance. As can be seen in Fig. S5, $\uparrow$ the PEI-40 vol\% BT/PVDF composite shows a higher $P_{\max }$ than the other bilayer composites with low $\mathrm{BaTiO}_{3}$ contents under the same charged electric field. However, its breakdown strength is obviously lower than those of the other bilayer composites. Consequently, it can only be charged to $280 \mathrm{kV} \mathrm{mm}^{-1}$ and the corresponding efficiency is lower than that of the other bilayer composites. In other words, higher $\mathrm{BaTiO}_{3}$ results in higher energy density but lower breakdown strength and lower efficiency. Furthermore, the (a)

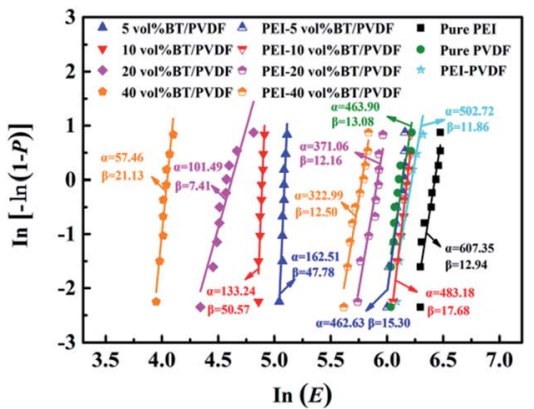

(b)

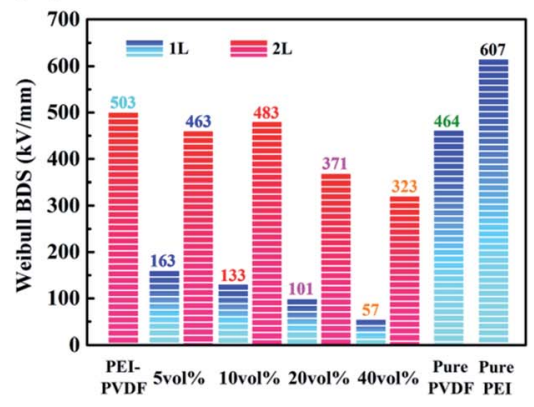

Fig. 4 (a) Weibull distribution plots and (b) characteristic breakdown strengths of the single layer and bilayer composites with different $\mathrm{BaTiO}_{3}$ loading fractions. 
(a)

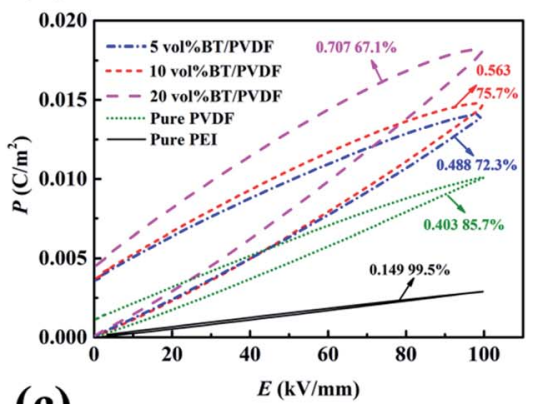

(c)

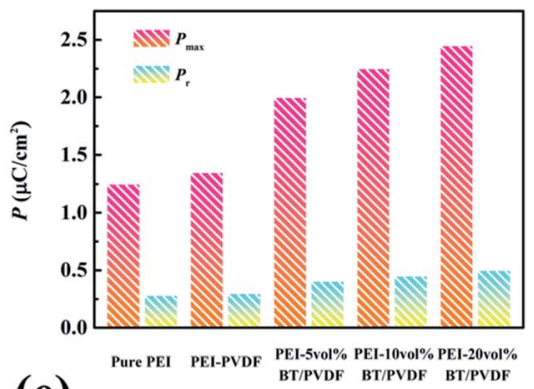

(e)

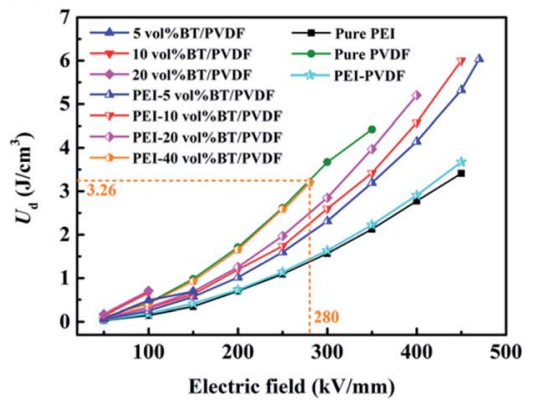

(b)

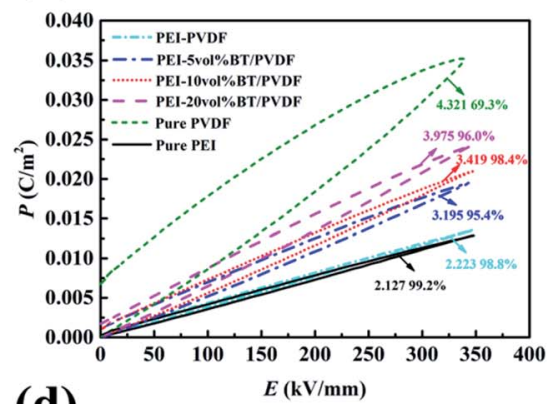

(d)

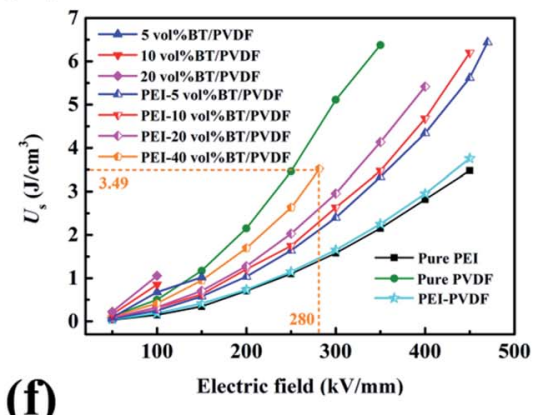

(f)

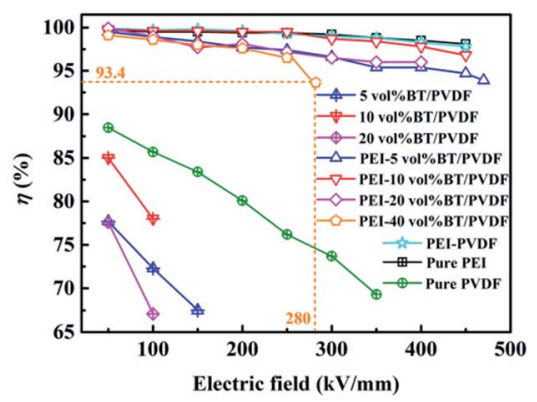

Fig. $5 \quad P-E$ loops of (a) single layer composites and (b) bilayer composites with different $\mathrm{BaTiO}_{3}$ loadings. (c) The maximum displacement $P_{\text {max }}$ and remnant displacement $P_{\mathrm{r}}$ of the pure PEI and bilayer composites at $350 \mathrm{kV} \mathrm{mm}^{-1}$. (d) The storage energy densities, (e) discharge energy densities and (f) discharge efficiencies of the single layer and bilayer composites under varied external electric fields.

stored energy densities $\left(U_{\mathrm{s}}\right)$ of the composites were derived from the $P-E$ loops (see Fig. $5 \mathrm{~d}$ and e, S3 and S4 $\dagger$ ) by integration of the area between the charge curve and the ordinate, while the discharge energy densities $\left(U_{\mathrm{d}}\right)$ were obtained by integrating the area between the discharge curve and the ordinate, and the discharge efficiencies $\eta$ were calculated using $\eta=U_{\mathrm{d}} / U_{\mathrm{s}}$. As illustrated in Fig. 5e, although the single layer BT/P(VDF-HFP) composites show higher $U_{\mathrm{d}}$ than the corresponding bilayer composites at low electric fields $\left(<200 \mathrm{kV} \mathrm{mm}^{-1}\right)$ as a result of their high permittivities, they cannot be used as energy storage materials under high electric fields owing to their low $E_{\mathrm{b}}$ as aforementioned. In contrast, the bilayer composites display superior $U_{\mathrm{d}}$ under high electric fields and their energy densities are much higher than that of the pure PEI. In particular, a high energy density of $\sim 6.0 \mathrm{~J} \mathrm{~cm}^{-3}$ under an electric field of $450 \mathrm{kV}$ $\mathrm{mm}^{-1}$ is achieved in the bilayer composite with $10 \mathrm{vol} \% \mathrm{BaTiO}_{3}$. The discharge efficiencies $\eta$ of the composites under different electric fields are illustrated in Fig. 5f. Apparently, ultrahigh $\eta$ $(>95 \%)$ values, which are much higher than those of the single layer pure $\mathrm{P}(\mathrm{VDF}-\mathrm{HFP})$ and $\mathrm{BT} / \mathrm{P}(\mathrm{VDF}-\mathrm{HFP})$ composites, are achieved. In the bilayer composites, the ultrahigh discharge efficiencies are mainly contributed by the introduction of the linear dielectric polymer PEI. On one hand, the aforementioned low volume fractions of ferroelectric phases, including $\mathrm{BaTiO}_{3}$ and $\mathrm{P}(\mathrm{VDF}-\mathrm{HFP})$, result in a low energy loss induced by ferroelectric polarizations, hence the efficiency is high. On the other hand, the excellent insulating properties of the PEI layer ensure high breakdown strengths of the bilayer composites which can effectively suppress the ohmic heat loss induced by leakage conductance and deliver a high discharge efficiency. Furthermore, the ultrahigh $\eta$ values are well maintained even under high electric fields up to $450 \mathrm{kV} \mathrm{mm}^{-1}$. In particular, the bilayer composite with $10 \mathrm{vol} \% \mathrm{BaTiO}_{3}$ exhibits an extremely high $\eta$ of $\sim 96.8 \%$ at $450 \mathrm{kV} \mathrm{mm}^{-1}$ which is almost as high as that of the pure PEI $(\eta \sim 98.2 \%)$. In contrast, the $\eta$ values of the single-layer $\mathrm{BT} / \mathrm{P}$ (VDF-HFP) composites become sharply deteriorated with increasing electric fields. As discussed above, the combination of linear and nonlinear dielectric polymers via constructing 


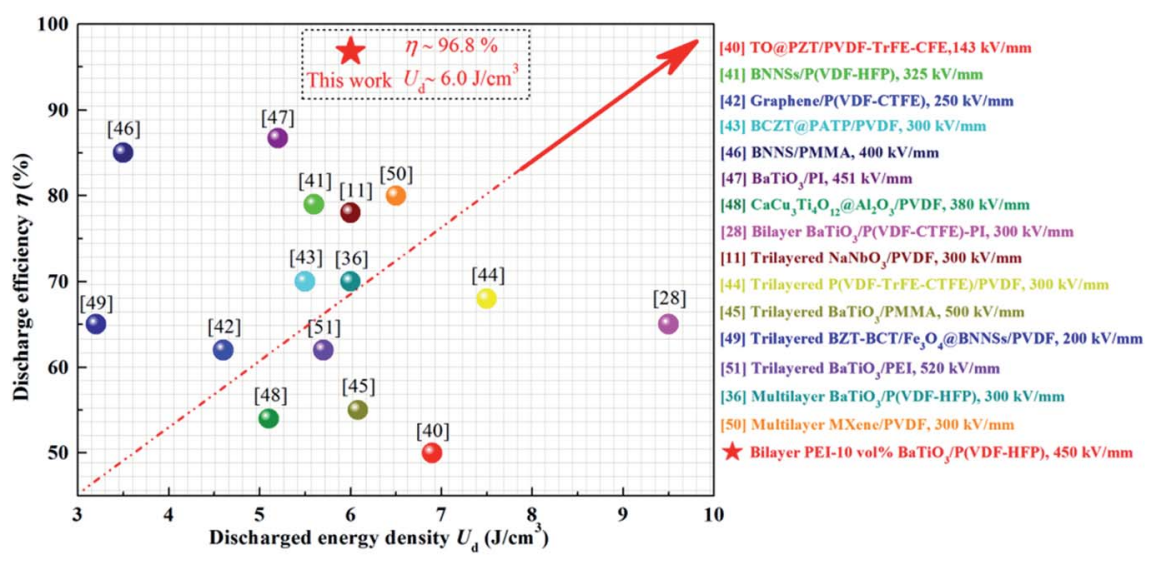

Fig. 6 Comparison of energy density and efficiency of this work (PEI-10 vol\% $\mathrm{BaTiO}_{3} / \mathrm{P}(\mathrm{VDF}-\mathrm{HFP})$ composite) and reported results.

bilayer composites gives rise to comprehensively enhanced energy-storage performances.

Fig. 6 compares the discharge efficiencies and energy densities of this work and of recently reported single layer, bilayer, trilayer and multilayer ( $>3$ layers) polymer composites from other research groups. ${ }^{\mathbf{4 0 - 5 1}}$ We can see that the composites using a linear dielectric polymer matrix (e.g., BNNS/PMMA composites in ref. 46 and $\mathrm{BaTiO}_{3} / \mathrm{PI}$ composites in ref. 47) display relatively higher discharge efficiencies $(>80 \%)$ than those composites based on nonlinear dielectric polymers (e.g., PVDF and its copolymers). It is worth noting that our bilayer PEI-10 vol\% $\mathrm{BaTiO}_{3} / \mathrm{P}(\mathrm{VDF}-\mathrm{HFP})$ composite exhibits a much higher discharge efficiency $(\sim 96.8 \%)$ than most of the so far reported polymer based dielectric composites. Meanwhile, a high energy density of $\sim 6 \mathrm{~J} \mathrm{~cm}^{-3}$ is also obtained. For a long time, the research on dielectric energy-storage materials was mainly targeted at solely achieving high energy density. In contrast, the discharge efficiency and loss that are key parameters in practical applications gained limited attention. The simultaneous realization of ultrahigh discharge efficiency and high energy density, low loss, and high breakdown strength makes these bilayer composites ideal candidates for applications in high-performance dielectric energy-storage devices.

\section{Conclusion}

In summary, a class of bilayer composites consisting of a pure polyetherimide layer and $\mathrm{BaTiO}_{3} / \mathrm{P}$ (VDF-HFP) composite layer were fabricated via solution casting and hot pressing. The bilayer composites show impressive improvements in breakdown strengths, i.e., $285 \%, 363 \%, 366 \%$ and $567 \%$ for composites with $5 \mathrm{vol} \%, 10 \mathrm{vol} \%, 20 \mathrm{vol} \%$ and $40 \mathrm{vol} \% \mathrm{BaTiO}_{3}$ particles, in comparison with their single layer counterparts, thus resulting in obviously improved $U_{\mathrm{d}}$. More notably, the bilayer composites exhibit ultrahigh discharge efficiencies over 95\%, which are much higher than most of the so far reported results, under external electric fields up to $400 \mathrm{kV} \mathrm{mm}^{-1}$. In particular, the bilayer composite with 10 vol\% $\mathrm{BaTiO}_{3}$ exhibits an ultrahigh discharge efficiency of $\sim 96.8 \%$ and a high energy density of $6 \mathrm{~J}$ $\mathrm{cm}^{-3}$ at $450 \mathrm{kV} \mathrm{mm}^{-1}$, along with a low loss tangent of $\sim 0.025$ @
$10 \mathrm{kHz}$. It is believed that, in the bilayer composites, the pure polyetherimide layer ensures high efficiency, high breakdown strength and low loss, while the $\mathrm{BaTiO}_{3} / \mathrm{P}(\mathrm{VDF}-\mathrm{HFP})$ composite layer offers high energy density. The synergy of the two layers brings about concurrently enhanced discharge efficiency and energy density. The excellent dielectric performances greatly promote the practical applications of these bilayer composites in advanced energy-storage devices. Hopefully, layer-structured composites with further improved dielectric properties may be developed via sufficiently exploring the synergic effects of the linear and nonlinear dielectric layers.

\section{Conflicts of interest}

There are no conflicts to declare.

\section{Acknowledgements}

The authors acknowledge the financial support of this work from the National Natural Science Foundation of China (51773187 and 51402271) and Foundation for Outstanding Young Scientist in Shandong Province (BS2014CL003).

\section{References}

1 X. Y. Huang, B. Sun, Y. K. Zhu, S. T. Li and P. K. Jiang, Prog. Mater. Sci., 2019, 100, 187-225.

2 H. Luo, X. F. Zhou, C. Ellingford, Y. Zhang, S. Chen, K. C. Zhou, D. Zhang, C. R. Bowen and C. Y. Wang, Chem. Soc. Rev., 2019, 48, 4424-4465.

3 H. Pan, F. Li, Y. Liu, Q. H. Zhang, M. Wang, S. Lan, Y. P. Zheng, J. Ma, L. Gu, Y. Shen, P. Yu, S. J. Zhang, L. Q. Chen, Y. H. Li and C. W. Nan, Science, 2019, 365, 578-582.

4 Z. P. Li, X. Y. Chen, C. Zhang, E. Baer, D. Langhe, M. Ponting, M. Brubaker, T. Hosking, R. P. Li, M. Fukuto and L. Zhu, ACS Appl. Polym. Mater., 2019, 1, 867-875.

5 Q. Y. Zhao, L. Yang, K. N. Chen, Y. Z. Ma, H. L. Ji, M. X. Shen, H. J. Huang, H. Y. He and J. H. Qiu, Nano Energy, 2019, 65, 104007. 
6 X. T. Zhu, J. Yang, D. Dastan, H. Garmestani, R. H. Fan and Z. C. Shi, Composites, Part A, 2019, 125, 105521.

7 J. Yang, X. T. Zhu, H. L. Wang, X. Wang, C. C. Hao, R. H. Fan, D. Dastan and Z. C. Shi, Composites, Part A, 2020, 131, 105814.

8 Y. N. Hao, X. H. Wang, K. Bi, J. M. Zhang, Y. H. Huang, L. W. Wu, P. Y. Zhao, K. Xu, M. Lei and L. T. Li, Nano Energy, 2017, 31, 49-56.

9 C. Zhang, Z. C. Shi, F. Mao, C. Q. Yang, J. Yang, X. T. Zhu and H. Zuo, J. Mater. Sci., 2018, 53, 1-13.

10 L. M. Yao, Z. B. Pan, J. W. Zhai, G. Z. Zhang, Z. Y. Liu and Y. H. Liu, Composites, Part A, 2018, 109, 48-54.

11 Z. B. Pan, B. H. Liu, J. W. Zhai, L. M. Yao, K. Yang and B. Shen, Nano Energy, 2017, 40, 587-595.

12 Z. C. Shi, S. G. Chen, R. H. Fan, X. A. Wang, X. Wang, Z. D. Zhang and K. Sun, J. Mater. Chem. C, 2014, 2, 6752.

13 X. Huang, X. Zhang, G. K. Ren, J. Y. Jiang, Z. K. Dan, Q. H. Zhang, X. Zhang, C. W. Nan and Y. Shen, J. Mater. Chem. A, 2019, 7, 15198.

14 P. T. Xie, Y. F. Li, Q. Hou, K. Y. Sui, C. Z. Liu, X. Y. Fu, J. X. Zhang, V. Murugadoss, J. C. Fan, Y. P. Wang, R. H. Fan and Z. H. Guo, J. Mater. Chem. C, 2020, 8, 30293039.

15 B. Xie, Y. W. Zhu, M. A. Marwat, S. J. Zhang, L. Zhang and H. B. Zhang, J. Mater. Chem. A, 2018, 6, 20356.

16 W. Y. Li, Z. Q. Song, J. Qian, Z. Y. Tan, H. Y. Chu, X. Y. Wu, W. Nie and X. H. Ran, Carbon, 2019, 141, 728-738.

17 K. Sun, J. N. Dong, Z. X. Wang, Z. Y. Wang, G. H. Fan, Q. Hou, L. Q. An, M. Y. Dong, R. H. Fan and Z. H. Guo, J. Phys. Chem. C, 2019, 123, 23635-23642.

18 L. Zhang, Z. Liu, X. Lu, G. Yang, X. Y. Zhang and Z. Y. Cheng, Nano Energy, 2016, 26, 550-557.

19 B. H. Fan, M. Y. Zhou, C. Zhang, D. L. He and J. B. Bai, Prog. Polym. Sci., 2019, 97, 101143.

20 M. F. Guo, J. Y. Jiang, Z. H. Shen, Y. H. Lin, C. W. Nan and Y. Shen, Mater. Today, 2019, 29, 49-67.

21 K. Bi, M. H. Bi, Y. N. Hao, W. Luo, Z. M. Cai, X. H. Wang and Y. H. Huang, Nano Energy, 2018, 51, 513-523.

22 G. Jian, M. R. Liu, C. Yan, F. Wu, B. Song, K. S. Moon and C. P. Wong, Nano Energy, 2019, 58, 419-426.

23 M. S. Zheng, Y. T. Zheng, J. W. Zha, Y. Yang, P. Han, Y. Q. Wen and Z. M. Dang, Nano Energy, 2018, 48, 144-151.

24 Y. Yang, Z. S. Gao, M. H. Yang, M. S. Zheng, D. R. Wang, J. W. Zha, Y. Q. Wen and Z. M. Dang, Nano Energy, 2019, 59, 363-371.

25 Z. Y. Wang, K. Sun, P. T. Xie, Q. Hou, Y. Liu, Q. L. Gu and R. H. Fan, Acta Mater., 2020, 185, 412-419.

26 Q. L. Fan, C. R. Ma, Y. Li, Z. S. Liang, S. Cheng, M. Y. Guo, Y. Z. Dai, C. S. Ma, L. Lu, W. Wang, L. H. Wang, X. J. Lou, M. Liu, H. Wang and C. L. Jia, Nano Energy, 2019, 62, 725733.

27 J. Y. Jiang, Z. H. Shen, J. F. Qian, Z. K. Dan, M. F. Guo, Y. He, Y. H. Lin, C. W. Nan, L. Q. Chen and Y. Shen, Nano Energy, 2019, 62, 220-229.

28 B. Xie, Q. Zhang, L. Zhang, Y. W. Zhu, X. Guo, P. Y. Fan and H. B. Zhang, Nano Energy, 2018, 54, 437-446.
29 Z. X. Sun, C. R. Ma, M. Liu, J. Cui, L. Lu, J. B. Lu, X. J. Lou, L. Jin, H. Wang and C. L. Jia, Adv. Mater., 2017, 29, 1604427.

30 Z. C. Shi, J. Wang, F. Mao, C. Q. Yang, C. Zhang and R. H. Fan, J. Mater. Chem. A, 2017, 5, 14575.

31 J. Wang, Z. C. Shi, F. Mao, S. G. Chen and X. Wang, ACS Appl. Mater. Interfaces, 2017, 9, 1793-1800.

32 J. Chen, Y. F. Wang, X. W. Xu, Q. B. Yuan, Y. J. Niu, Q. Wang and H. Wang, J. Mater. Chem. A, 2019, 7, 3729.

33 Y. F. Wang, L. X. Wang, Q. B. Yuan, J. Chen, Y. J. Niu, X. W. Xu, Y. T. Cheng, B. Yao, Q. Wang and H. Wang, Nano Energy, 2018, 44, 364-370.

34 C. Jie, Y. F. Wang, Q. B. Yuan, X. W. Xu, Y. J. Niu, Q. Wang and H. Wang, Nano Energy, 2018, 54, 288-296.

35 Y. F. Wang, J. Chen, Y. Li, Y. J. Niu, Q. Wang and H. Wang, J. Mater. Chem. A, 2019, 7, 2965.

36 Y. F. Wang, Y. Li, L. X. Wang, Q. B. Yuan, J. Chen, Y. J. Niu, X. W. Xu, Q. Wang and H. Wang, Energy Storage Materials, 2020, 24, 626-634.

37 C. Zhang, Z. C. Shi, F. Mao, C. Q. Yang, X. T. Zhu, J. Yang, H. Zuo and R. H. Fan, ACS Appl. Mater. Interfaces, 2018, 10, 26713-26722.

38 Y. Feng, Y. H. Zhou, T. D. Zhang, C. H. Zhang, Y. Q. Zhang, Y. Zhang, Q. G. Chen and Q. G. Chi, Energy Storage Materials, 2020, 25, 180-192.

39 Y. Zhang, C. H. Zhang, Y. Feng, T. D. Zhang, Q. G. Chen, Q. G. Chi, L. Z. Liu, X. Wang and Q. Q. Lei, Nano Energy, 2019, 64, 104195.

40 D. Zhang, W. W. Liu, R. Guo, K. C. Zhou and H. Luo, Adv. Sci., 2018, 5, 1700512.

41 H. J. Ye, T. M. Lu, C. F. Xu, M. Q. Zhong and L. X. Xu, Nanotechnology, 2018, 29, 095702.

42 H. J. Ye, X. H. Zhang, C. F. Xu, B. Han and L. X. Xu, J. Mater. Chem. C, 2018, 6, 11144-11155.

43 Z. B. Pan, L. M. Yao, J. W. Zhai, H. T. Wang and B. Shen, ACS Appl. Mater. Interfaces, 2017, 9, 14337-14346.

44 L. Wang, H. Luo, X. F. Zhou, X. Yuan, K. C. Zhou and D. Zhang, Composites, Part A, 2019, 117, 369-376.

45 M. A. Marwat, B. Xie, Y. W. Zhu, P. Y. Fan, W. G. Ma, H. M. Liu, M. Ashtar, J. Z. Xiao, D. Salamon, C. Samater and H. B. Zhang, Composites, Part A, 2019, 121, 115-122.

46 F. H. Liu, Q. Li, Z. Y. Li, Y. Liu, L. J. Dong, C. X. Xiong and Q. Wang, Compos. Sci. Technol., 2017, 142, 139-144.

47 W. D. Sun, X. J. Lu, J. Y. Jiang, X. Zhang, P. H. Hu, M. Li, Y. H. Lin, C. W. Nan and Y. Shen, J. Appl. Phys., 2017, 121, 244101.

48 Q. G. Chi, X. B. Wang, C. H. Zhang, Q. G. Chen, M. H. Chen, T. D. Zhang, L. Gao, Y. Zhang, Y. Cui, X. Wang and Q. Q. Lei, ACS Sustainable Chem. Eng., 2018, 6, 8641-8649.

49 Y. Zhang, T. D. Zhang, L. Z. Liu, Q. G. Chi, C. H. Zhang, Q. G. Chen, Y. Cui, X. Wang and Q. Q. Lei, J. Phys. Chem. C, 2018, 122, 1500-1512.

50 W. Y. Li, Z. Q. Song, J. M. Zhong, J. Qian, Z. Y. Tan, X. Y. Wu, H. Y. Chu, W. Nie and X. H. Ran, J. Mater. Chem. C, 2019, 7, 10371.

51 M. A. Marwat, B. Xie, Y. W. Zhu, P. Y. Fan, K. Liu, M. Shen, M. Ashtar, S. Kongparakul, C. Samater and H. B. Zhang, Colloids Surf., A, 2019, 581, 123802. 